\title{
Comorbidity and glycemia control among patients with type 2 diabetes in primary care
}

\author{
Catherine Hudon ${ }^{1,3}$ \\ Martin Fortin ${ }^{1,3}$ \\ Marie-France Dubois ${ }^{2}$ \\ José Almirall \\ 'Department of Family Medicine, \\ ${ }^{2}$ Department of Community Health \\ Sciences, Sherbrooke University, \\ Sherbrooke, Quebec, Canada; ${ }^{3}$ Centre \\ de Santé et de Services Sociaux de \\ Chicoutimi, Quebec, Canada
}

\begin{abstract}
Reports on the relationship between comorbidity and glycemia control in diabetic patients are conflicting and the method of measuring comorbidity varies widely among studies. The aim of the present study was to evaluate the relationship between diabetes control and comorbidity, taking into account all comorbidities and their severity, in a primary care setting. We performed a retrospective descriptive study based on chart review of 96 randomly selected type 2 diabetic patients. Comorbidity was measured with the cumulative illness rating scale (CIRS), an exhaustive comorbidity index. Diabetes was considered as controlled if the mean value of two measurements of glycosylated hemoglobin $\mathrm{A}\left(\mathrm{HbA}_{1 \mathrm{c}}\right)$ was less than $7 \%$. Taking diabetes control as the dependent variable, its relationship with the CIRS score, age, sex, diabetes duration, and diabetes-related complications was explored. Diabetes control was not significantly related with the CIRS score, age, sex or diabetes severity. Diabetes duration was the only variable significantly related to diabetes control. Our study suggests that comorbidity measured with the CIRS in patients with type 2 diabetes is not a factor that prevents the achievement of a good glycemia control.
\end{abstract}

Keywords: glycemia control, type 2 diabetes mellitus, comorbidity, primary care

\section{Introduction}

Diabetes mellitus is one of the most challenging chronic diseases (King et al 1998; Lipscombe and Hux 2007) associated with significant morbidity and mortality (Baker et al 2008; Pinto et al 2008). In order to avoid or delay microvascular complications, a strict metabolic control of blood glucose is recommended (UKPDS 1998; LeRoith and Smith 2005; Vermeire et al 2005). Measure of glycosylated hemoglobin $\mathrm{A}\left(\mathrm{HbA}_{1 \mathrm{c}}\right)$ is used to evaluate glycemia control. For the majority of diabetics, glycemia control is considered attained when $\mathrm{HbA}_{1 \mathrm{c}}$ levels are less than 7\% (Harris and Lank 2004; Anonymous 2008).

Many patients do not achieve optimal glycemia control (Saaddine et al 2002; Putzer et al 2004). Longer diabetes duration and presence of complications are both associated with poor glycemia control (Druss et al 2001; Putzer et al 2004; Weiner and Long 2004; Egede 2005; Kerr et al 2007; Kivimaki et al 2007; Parchman et al 2007; Suh et al 2008). The majority of diabetic patients have at least one comorbid chronic condition (Druss et al 2001; Egede 2005; Kivimaki et al 2007), a problem that may also have an impact on glycemia control. Indeed, competing situations may occur in the treatment of patients (Parchman et al 2007), and the presence of several symptomatic comorbidities may influence patients' self management (Kerr et al 2007). However, reports on the relationship between comorbidity and glycemia control are conflicting. In some cases, evidence suggests that comorbidity is associated with lower glycemia control (Zhang et al 2000; Weiner and Long 2004; Suh et al 2008), whereas other data argue against any association (El-Kebbi et al 2001). These conflicting results may be explained by the use of different methods to measure comorbidity. In two studies, 
the Charlson comorbidity index was used (Zhang et al 2000; Weiner and Long 2004). However, this index was originally developed to predict mortality risk and includes a limited list of diseases. Another study used the chronic disease score (CDS) (El-Kebbi et al 2001), a comorbidity measure based on sex, age, and drugs prescribed to the patient. The remaining study took into account a limited number of conditions traditionally known as diabetes complications (Suh et al 2008).

Uncontrolled diabetes may result in complications such as nephropathy, renal failure, hypertension, congestive heart failure and coronary disease (ADA 2008). Therefore, any study on a possible association between glycemia control and comorbidity, in which the measure of comorbidity would only include such chronic conditions would be biased towards finding an association. Furthermore, in addition to the type of comorbidity, its severity may either cause poor glycemia control or be a consequence of it.

Since many diabetic patients have comorbidity (Druss et al 2001; Egede 2005; Kivimaki et al 2007), it is important to determine if comorbidity increases the difficulty to manage the disease and to achieve good glycemia control. Therefore, the aim of the present study was to evaluate the relationship between diabetes control and comorbidity using a comprehensive comorbidity index in a primary care setting.

\section{Methods}

We performed a retrospective descriptive study based on chart review at the family medicine unit (FMU) of the Centre de Santé et de Services Sociaux de Chicoutimi, Quebec, Canada. The study was approved by the institutional ethics committee.

Using the electronic laboratory database available, we performed a random selection of patients with type 2 diabetes who had at least two measurements of $\mathrm{HbA}_{1 \mathrm{c}}$ levels between January 1st, 2004 and September 1st, 2006. Patients diagnosed with diabetes less than a year before the last measurement of $\mathrm{HbA}_{1 \mathrm{c}}$ and those under the care of the researchers involved in this study were excluded.

Three trained nurses extracted data from patients' paper charts. Data extracted from the charts included sex, age, the last two measures of $\mathrm{HbA}_{1 \mathrm{c}}$, the presence or absence of diabetes-related complications, time elapsed since the diagnosis of diabetes (diabetes duration at the moment of the second measurement of $\mathrm{HbA}_{1 \mathrm{c}}$ ) as well as information about the patient's chronic conditions necessary to score the cumulative illness rating scale (CIRS) (Linn et al 1968; Fortin et al 2005b, 2006a, 2006b). The three nurses, blinded to the objectives of the study, had received an half-day training in CIRS scoring prior to the study.

The CIRS is a measure of comorbidity that takes into account all medical conditions and includes the notion of severity (Linn et al 1968; Fortin et al 2005b, 2006a, $2006 b)$. It has been validated in primary care as a tool for quantifying comorbidity (Hudon et al 2005, 2007). The CIRS uses a scoring system that encompasses 14 anatomical domains (cardiac, vascular, hematological, respiratory, ophthalmologic-otorhinolaryngologic, upper gastrointestinal, lower gastrointestinal, hepatic-pancreatic, renal, genitourinary, musculoskeletal-tegument, neurological, endocrine-metabolic-breast, and psychiatric) and assigns a value from 0 (no condition in this domain) to 4 (extremely severe problem) to determine a severity score for each domain. In the case of multiple conditions affecting a particular domain, the highest score is given to the domain. The global score is the sum of each domain's score. To use the CIRS as a measure of comorbidity for diabetes, this disease was not included in the global score of each patient.

Study groups were defined with a categorical variable (controlled/uncontrolled diabetes). Diabetes was considered as controlled if the mean value of the two measurements of $\mathrm{HbA}_{1 \mathrm{c}}$ levels was less than 7\%; mean values equal or greater than $7 \%$ were considered as uncontrolled diabetes (Harris and Lank 2004; Anonymous 2008).

\section{Data analysis}

The differences between patients with controlled and uncontrolled diabetes were tested using the $t$-test for continuous variables such as CIRS score, age and diabetes duration, and the Chi-square test for categorical variables like sex and diabetes-related complications. Then, variables with $P<0.1$ were used as covariables in a multivariable logistic regression model to measure the association between diabetes control (dependant variable) and the CIRS score (independent variable).

According to various studies, the percentage of patients controlling their diabetes varies from 33\% to 55\% (Weiner and Long 2004; Meduru et al 2007; Suh et al 2008). When we estimated the sample size, we used the worst case scenario and anticipated a 2-to-1 ratio when comparing subjects with uncontrolled diabetes to subjects with controlled diabetes. A total sample size of 75 subjects was needed in order to achieve $80 \%$ statistical power, to detect a difference of 4 points on the CIRS with a standard deviation of 5.64 (based on a previous study) (Fortin et al 2005a), with a two-sided t-test at the 5\% significance level (nQuery Advisor 6.01). 
We increased this sample size by $30 \%$ to account for possible missing data in the charts and to allow controlling for 2 to 3 variables in the logistic regression model (requiring at least 30 subjects in the smallest group).

Analyses were made with SPSS software (version 13.0; SPSS Inc., Chicago, IL) and a $P$ value $<0.05$ was considered statistically significant.

\section{Results}

Ninety-six charts were reviewed for this study. Patients' characteristics and the two groups of diabetic patients are presented in Table 1. Statistical results from the univariate analyses performed to measure the associations between diabetes control and the patients' characteristics are indicated in Table 1.

Diabetes control was not significantly related with the CIRS score, age, sex, or diabetes-related complications. Diabetes control was however significantly related to diabetes duration. A logistic regression model was thus used to measure the association between diabetes control and the CIRS, by including the diabetes duration as a covariable. The relation was still not significant (adjusted odds ratio $=0.889$; 95\% confidence interval: $0.736-1.074 ; p=0.223$ ).

\section{Discussion}

The results of this study suggest that in patients with type 2 diabetes mellitus, the presence of comorbidity measured with the CIRS, is not related to glycemia control defined by $\mathrm{HbA}_{1 \mathrm{c}}$ levels. The finding that glycemia control is associated with diabetes duration is similar to that of previous reports (Leelawattana et al 2006; Shim et al 2006; Tascona et al 2006; Heisler et al 2007; Lopez Stewart et al 2007; Tien et al 2008; Tikellis et al 2008).

To our knowledge, this is the first study that used the CIRS to measure comorbidity in order to assess the relationship between comorbidity and glycemia control in type 2 diabetic patients. The CIRS score takes into account all comorbidities as well as their severity, and provides a good assessment of other chronic diseases that may coexist with diabetes mellitus, being or not etiologically associated with it. Compared to our comorbidity measuring method, previous studies on a possible association between comorbidity condition and glycemia control included a limited number of diseases (Zhang et al 2000; Weiner and Long 2004; Suh et al 2008) or diseases associated to diabetes exclusively (Suh et al 2008). In these studies, the presence of comorbidities was found to be related to glycemia control. Our finding that the presence of comorbidities is not associated with glycemia control supports the conclusion of another study which also used a more comprehensive measurement of comorbidity, the chronic disease score (CDS) (El-Kebbi et al 2001).

It has been reported that depending on the type of comorbid condition they have, diabetic patients are more or less likely to achieve $\mathrm{HbA}_{1 \mathrm{c}}$ levels under 7\% (Meduru et al 2007). Therefore, when $\mathrm{HbA}_{1 \mathrm{c}}$ less than $7 \%$ threshold is used as a performance measure for diabetes quality of care, assessment of an association between glycemia control and comorbidity may be influenced by the type of comorbid conditions. In fact, the likelihood of finding an association between poor glycemia control and comorbidity in patients with diabetes-related complications would be higher than in patients without this type of complications (Meduru et al 2007). However, our study suggests that the presence of multiple chronic conditions is not a factor limiting the achievement of a good control of glycemia, even after taking into account the severity of the coexistent diseases. It has been reported that services received by diabetic patients do not differ based on comorbid illness burden (Halanych et al 2007), however diabetic patients with elevated $\mathrm{HbA}_{1 \mathrm{c}}$ receive a closer

Table I Comparison of patients with controlled or uncontrolled diabetes

\begin{tabular}{|c|c|c|c|}
\hline Characteristic* & $\begin{array}{l}\text { Controlled diabetes }\left(\mathrm{HbA}_{\mathrm{lc}}<7 \%\right) \\
(\mathrm{n}=66)\end{array}$ & $\begin{array}{l}\text { Uncontrolled diabetes }\left(\mathrm{HbA}_{\mathrm{Ic}} \geq 7 \%\right) \\
(\mathrm{n}=30)\end{array}$ & $P$ \\
\hline Males, n (\%) & $30.0(45.0)$ & $17.0(57.0)$ & $0.340^{\dagger}$ \\
\hline Age, years & $67.8(10.6)$ & $65.2(12.4)$ & $0.290 * *$ \\
\hline \multirow[t]{2}{*}{ Diabetes duration, years } & $6.7(4.5)$ & II.3 (7.0) & $0.003 * *$ \\
\hline & $(\mathrm{n}=34)^{\S}$ & $(n=23)^{\S}$ & \\
\hline CIRS score & $9.0(4.0)$ & $8.9(3.7)$ & $0.850 * *$ \\
\hline $\mathrm{HbA}_{\mathrm{Ic}}{ }^{* * *}, \%$ & $6.1(0.4)$ & $8.1(1.2)$ & $0.007 * *$ \\
\hline Diabetes-related complications, n (\%) & $22.0(33.0)$ & $15.0(50.0)$ & $0.120^{\dagger}$ \\
\hline
\end{tabular}

Notes: *Unless otherwise indicated, data are given as mean (SD); ${ }^{+P}$-value was based on Chi-square test; ${ }^{* *} P$-value was based on $t$-test; ${ }^{* * *}$ Average of two measurements; ${ }^{\S}$ Number of patients for whom information on diabetes duration was available.

Abbreviations: $\mathrm{HbA}_{1 \mathrm{c}}$, glycosylated hemoglobin; CIRS, cumulative illness rating scale. 
attention in primary care encounters (Parchman et al 2007). Therefore, in the presence of multiple comorbid conditions, diabetic patients may receive better care. As a matter of fact, it has been reported that vulnerable elders with multiple chronic conditions, including diabetes, receive better overall quality of care (Min et al 2007), and that the quality of care improves as a patient's number of chronic conditions increases (Higashi et al 2007).

\section{Limitations}

It could have been preferable to use a mean value of all $\mathrm{HbA}_{\mathrm{lc}}$ over two years rather than using only the most recent two $\mathrm{HbA}_{1 \mathrm{c}}$ measures. However, given data abstraction techniques, it was not feasible to utilize additional values. Since we performed a retrospective study, one limitation of our method is that different variables such as the time elapsed between the two measurements of $\mathrm{HbA}_{1 \mathrm{c}}$ could not be controlled. Furthermore, in our sample, $69 \%$ of the patients had a good glycemia control (Table 1), a higher proportion compared to that in other studies (33\%-55\%) (Weiner and Long 2004; Meduru et al 2007; Suh et al 2008). The fact that our institution is a teaching hospital with a FMU may have influenced the quality of care provided, and biased the results towards a better glycemia control. There is also the possibility that a number of patients with more severe diabetes are followed up in specialized clinics and were therefore underrepresented in our sample. Another limitation of the study is that the patients were recruited from only one setting and therefore extrapolation of our results to other settings is more difficult to make.

Lack of statistical power could be an issue in the assessment of the relationship between diabetes-related complications and glycemia control. Given that the proportion of complications is around $50 \%$ when diabetes is not controlled, the two group chi-square test at the 0.05 level had a $34 \%$ statistical power to detect an odds ratio of 0.5 with sample sizes of 30 (uncontrolled diabetes) and 66 (controlled diabetes) (nQuery Advisor 6.01).

Despite these limitations, our study is strengthened by the use of a comprehensive comorbidity index to measure comorbidity that takes into account disease severity, a random patients' selection and the mean of two $\mathrm{HbA}_{1 \mathrm{c}}$ measurements instead of a single measurement.

\section{Conclusion}

Our study suggests that comorbidity, measured with an exhaustive index, is not a factor that prevents the achievement of a good glycemia control in patients with type 2 diabetes.
Further studies are needed to extend these results to other settings and to evaluate the impact of different types of medical conditions on glycemia control.

\section{Acknowledgments}

We would like to acknowledge the contribution from the Centre de Santé et de Services Sociaux that funded the Chaire de recherche sur les maladies chroniques en soins de première ligne, hosted by the University of Sherbrooke. We also thank Mrs Tania Fayad for her editorial assistance with the preparation and revision of this paper. We report no conflicts of interest in this work.

\section{References}

[ADA] American Diabetes Association. 2008. Type 2 diabetes complications [online]. Accessed Jun 26, 2008. URL: http://www.diabetes.org/type2-diabetes/complications.jsp.

Anonymous. 2008. Executive summary: Standards of medical care in diabetes - 2008. Diabetes Care, 31:S5-S11.

Baker ST, Chiang CY, Zajac JD, et al. 2008. Outcomes for general medical inpatients with diabetes mellitus and new hyperglycaemia. Med J Aust, 188:340-3.

Druss BG, Marcus SC, Olfson M, et al. 2001. Comparing the national economic burden of five chronic conditions. Health Aff, 20:233-41.

Egede LE. 2005. Effect of comorbid chronic diseases on prevalence and odds of depression in adults with diabetes. Psychosom Med, 67:46-51.

El-Kebbi IM, Ziemer DC, Cook CB, et al. 2001. Comorbidity and glycemic control in patients with type 2 diabetes. Arch Intern Med, 161:1295-300.

Fortin M, Bravo G, Hudon C, et al. 2006a. Relationship between multimorbidity and health-related quality of life of patients in primary care. Qual Life Res, 15:83-91.

Fortin M, Bravo G, Hudon C, et al. 2006b. Relationship between psychological distress and multimorbidity of patients in family practice. Ann Fam Med, 4:417-22.

Fortin M, Bravo G, Hudon C, et al. 2005a. Prevalence of multimorbidity among adults seen in family practice. Ann Fam Med, 3:223-8.

Fortin M, Hudon C, Dubois M-F, et al. 2005b. Comparative assessment of three different indices of multimorbidity for studies on health-related quality of life. Health Qual Life Outcomes, 3:74

Halanych JH, Safford MM, Keys WC, et al. 2007. Burden of comorbid medical conditions and quality of diabetes care. Diabetes Care, 30:2999-3004.

Harris SB, Lank CN. 2004. Recommendations from the Canadian Diabetes Association. 2003 guidelines for prevention and management of diabetes and related cardiovascular risk factors. Can Fam Physician, 50:425-33.

Heisler M, Faul JD, Hayward RA, et al. 2007. Mechanisms for racial and ethnic disparities in glycemic control in middle-aged and older Americans in the health and retirement study. Arch Intern Med, 167:1853-60.

Higashi T, Wenger NS, Adams JL, et al. 2007. Relationship between number of medical conditions and quality of care. $N$ Engl $J$ Med, 356:2496-504

Hudon C, Fortin M, Soubhi H. 2007. Abbreviated guidelines for scoring the Cumulative Ilnness Rating Scale (CIRS) in family practice. J Clin Epidemiol, 60:212.

Hudon C, Fortin M, Vanasse A. 2005. Cumulative Illness Rating Scale was a reliable and valid index in a family practice context. $J$ Clin Epidemiol, 58:603-8.

Kerr EA, Heisler M, Krein SL, et al. 2007. Beyond comorbidity counts: how do comorbidity type and severity influence diabetes patients' treatment priorities and self-management? J Gen Intern Med, 22:1635-40. 
King H, Aubert RE, Herman WH. 1998. Global burden of diabetes, 1995-2025: prevalence, numerical estimates, and projections. Diabetes Care, 21:1414-31.

Kivimaki M, Vahtera J, Pentti J, et al. 2007. Increased sickness absence in diabetic employees: what is the role of co-morbid conditions? Diabet Med, 24:1043-8.

Leelawattana R, Pratipanawatr T, Bunnag P, et al. 2006. Thailand diabetes registry project: prevalence of vascular complications in long-standing type 2 diabetes. J Med Assoc Thai, 89(Suppl 1):S54-9.

LeRoith D, Smith DO. 2005. Monitoring glycemic control: the cornerstone of diabetes care. Clin Ther, 27:1489-99.

Linn BS, Linn MW, Gurel L. 1968. Cumulative illness rating scale. $J$ Am Geriatr Soc, 16:622-6.

Lipscombe LL, Hux JE. 2007. Trends in diabetes prevalence, incidence, and mortality in Ontario, Canada 1995-2005: a population-based study. Lancet, 369:750-6.

Lopez Stewart G, Tambascia M, Rosas Guzman J, et al. 2007. Control of type 2 diabetes mellitus among general practitioners in private practice in nine countries of Latin America. Rev Panam Salud Publica, 22:12-20.

Meduru P, Helmer D, Rajan M, et al. 2007. Chronic illness with complexity: implications for performance measurement of optimal glycemic control. J Gen Intern Med, 22(Suppl 3):408-18.

Min LC, Wenger NS, Fung C, et al. 2007. Multimorbidity is associated with better quality of care among vulnerable elders. Med Care, 45:480-8.

Parchman ML, Pugh JA, Romero RL, et al. 2007. Competing demands or clinical inertia: the case of elevated glycosylated hemoglobin. Ann Fam Med, 5:196-201.

Pinto A, Tuttolomondo A, Di Raimondo D, et al. 2008. Cardiovascular risk profile and morbidity in subjects affected by type 2 diabetes mellitus with and without diabetic foot. Metabolism, 57:676-82.

Putzer GJ, Ramirez AM, Sneed K, et al. 2004. Prevalence of patients with type 2 diabetes mellitus reaching the American Diabetes Association's target guidelines in a university primary care setting. South Med J, 97:145-8.
Saaddine JB, Engelgau MM, Beckles GL, et al. 2002. A diabetes report card for the United States: quality of care in the 1990s. Ann Intern Med, 136:565-74.

Shim WS, Kim SK, Kim HJ, et al. 2006. Decrement of postprandial insulin secretion determines the progressive nature of type-2 diabetes. Eur $J$ Endocrinol, 155:615-22.

Suh DC, Kim CM, Choi IS, et al. 2008. Comorbid conditions and glycemic control in elderly patients with type 2 diabetes mellitus, 1988 to 1994 to 1999 to 2004. J Am Geriatr Soc, 56:484-92.

Tascona DJ, Morton AR, Toffelmire EB, et al. 2006. Adequacy of glycemic control in hemodialysis patients with diabetes. Diabetes Care, 29:2247-51.

Tien KJ, Hung HC, Hsiao JY, et al. 2008. Effectiveness of comprehensive diabetes care program in Taiwanese with type 2 diabetes. Diabetes Res Clin Pract, 79:276-83.

Tikellis G, Wang S, Wong N, et al. 2008. Poor metabolic and blood pressure control in patients with diabetic retinopathy attending a tertiary ophthalmic hospital in Australia. Diabetes Res Clin Pract, 80:208-12.

[UKPDS] UK Prospective Diabetes Study Group. 1998. Intensive bloodglucose control with sulfonylurea or insulin compared with conventional treatment and risk of complications in patients with type 2 diabetes (UKPDS 33) UK Prospective Diabetes Study (UKPDS) Group. Lancet, 352:837-53.

Vermeire E, Wens J, Van Royen P, et al. 2005. Interventions for improving adherence to treatment recommendations in people with type 2 diabetes mellitus. Cochrane Database Syst Rev, 1:CD003638.

Weiner M, Long J. 2004. Cross-sectional versus longitudinal performance assessments in the management of diabetes. Med Care, 42(2 Suppl):II34-9.

Zhang Q, Safford M, Ottenweller J, et al. 2000. Performance status of health care facilities changes with risk adjustment of $\mathrm{HbAl}$. Diabetes Care, 23:919-27. 
